\title{
IDENTITIES AND CONGRUENCES INVOLVING THE GEOMETRIC POLYNOMIALS
}

\author{
MILOUD MIHOUBI AND SAID TAHARBOUCHET
}

Received 13 January, 2018

\begin{abstract}
In this paper, we investigate the umbral representation of the geometric polynomials $\mathbf{w}_{\mathbf{x}}^{n}:=w_{n}(x)$ to derive some properties involving these polynomials. Furthermore, for any prime number $p$ and any polynomial $f$ with integer coefficients, we show $\left(f\left(\mathbf{w}_{\mathbf{x}}\right)\right)^{p} \equiv f\left(\mathbf{w}_{\mathbf{x}}\right)$ (mod $p$ ) and we give other curious congruences.
\end{abstract}

2010 Mathematics Subject Classification: 05A18; 05A40; 11A07

Keywords: geometric umbra, geometric polynomials, identities, congruences

\section{INTRODUCTION}

The geometric numbers are quantities arising from enumerative combinatorics and have nice number-theoretic properties. In combinatorics, the $n$-th geometric number (named also the $n$-th ordered Bell number) counts the number of ways to partition the set $[n]:=\{1, \ldots, n\}$ into ordered subsets $[2,3,6]$. The geometric polynomials are defined by $w_{n}(x)=\sum_{k=0}^{n}\left\{\begin{array}{l}n \\ k\end{array}\right\} k ! x^{k}$ and satisfy the recurrence relation $(x+1) w_{n}(x)=x \sum_{j=0}^{n}\left(\begin{array}{l}n \\ j\end{array}\right) w_{j}(x), n \geq 1$, [9], where $\left\{\begin{array}{l}n \\ k\end{array}\right\}$ is the $(n, k)$-th Stirling number of the second kind $[2,26]$. These polynomials have attracted attention from many researchers, see for instance $[9,10,15-17]$. For $x=1$ we obtain the geometric numbers $w_{n}:=w_{n}(1)=\sum_{k=0}^{n}\left\{\begin{array}{l}n \\ k\end{array}\right\} k$ !, for more information about these numbers, see $[6-8,11,12,14,28,29]$. More generally, let $w_{n}(x ; r, s)$ be the $n$-th $(r, s)$-geometric polynomial defined by

$$
w_{n}(x ; r, s)=\sum_{k=0}^{n}\left\{\begin{array}{l}
n+r \\
k+r
\end{array}\right\}_{r}(k+s) ! x^{k} .
$$

This polynomial generalizes the geometric polynomial $w_{n}(x)=w_{n}(x ; 0,0)$ and the polynomial $w_{n}(x ; r, r)$ introduced by Mezô [18]. Here, $\left\{\begin{array}{l}n \\ k\end{array}\right\}_{r}$ denotes the $(n, k)$-th $r$-Stirling number of the second kind [4]. One can see easily that

$$
\begin{aligned}
& w_{0}(x ; r, s)=s ! \\
& w_{1}(x ; r, s)=s !(r+(s+1) x),
\end{aligned}
$$




$$
w_{2}(x, r, s)=s !\left(r^{2}+(2 r+1)(s+1) x+(s+1)(s+2) x^{2}\right) .
$$

We note that this generalization can be viewed as a particular case of that defined by Kargin et al. [16]. As it shown below, these polynomials are also linked to the absolute $r$-Stirling numbers of first kind denoted by $\left[\begin{array}{l}n \\ k\end{array}\right]_{r}$.

Recall that the $r$-Stirling numbers can be defined by $[4,26]$

$$
(x)_{n}=\sum_{k=0}^{n}(-1)^{n-k}\left[\begin{array}{l}
n+r \\
k+r
\end{array}\right]_{r}(x+r)^{k} \text { and }(x+r)^{n}=\sum_{k=0}^{n}\left\{\begin{array}{l}
n+r \\
k+r
\end{array}\right\}_{r}(x)_{k},
$$

where $(\alpha)_{n}=\alpha \cdots(\alpha-n+1)$ if $n \geq 1,(\alpha)_{0}=1$.

This work is motivated by application of the umbral calculus method to determine identities and congruences involving Bell numbers and polynomials in the works of Gessel [13], Sun et al. [27], Mezô et al. [19] and Benyattou et al. [1]. In this paper, we will talk about identities and congruences involving the $(r, s)$-geometric polynomials based on the geometric umbra defined by $\mathbf{w}_{\mathbf{x}}^{n}:=w_{n}(x)$. For more information about umbral calculus, see [5, 13,22-25].

\section{IDENTITIES INVOLVING THE $(r, s)$-GEOMETRIC POLYNOMIALS}

The above recurrence relation is equivalent to $(x+1) \mathbf{w}_{\mathbf{x}}^{n}=x\left(\mathbf{w}_{\mathbf{x}}+1\right)^{n}, n \geq 1$. Furthermore, we have

Proposition 1. Let $f$ be a polynomial and $r$, be non-negative integers. Then

$$
\begin{aligned}
& (x+1) f\left(\mathbf{w}_{\mathbf{x}}+r\right)=x f\left(\mathbf{w}_{\mathbf{x}}+r+1\right)+f(r), \\
& \left(\mathbf{w}_{\mathbf{x}}+r\right)_{n+r}=(n+r) ! x^{n}(x+1)^{r}, \\
& \left(\mathbf{w}_{\mathbf{x}}+r-s\right)^{n}\left(\mathbf{w}_{\mathbf{x}}\right)_{s}=x^{s} w_{n}(x ; r, s), \\
& \left(\mathbf{w}_{\mathbf{x}}+r\right)^{n}\left(\mathbf{w}_{\mathbf{x}}+s\right)_{s}=(x+1)^{s} w_{n}(x ; r, s) .
\end{aligned}
$$

Proof. It suffices to show the first identity for $f(x)=x^{n}$. For $r=0$ we have $(x+1) \mathbf{w}_{\mathbf{x}}^{n}-x\left(\mathbf{w}_{\mathbf{x}}+1\right)^{n}=\delta_{(n=0)}$. Assume it is true for $r-1$, then if we set

$$
h_{n}(r):=(x+1)\left(\mathbf{w}_{\mathbf{x}}+r\right)^{n}-x\left(\mathbf{w}_{\mathbf{x}}+r+1\right)^{n}
$$

we obtain $h_{n}(r)=\sum_{j=0}^{n}\left(\begin{array}{c}n \\ j\end{array}\right) h_{j}(r-1)=\sum_{j=0}^{n}\left(\begin{array}{c}n \\ j\end{array}\right)(r-1)^{j}=r^{n}$, which concludes the induction step. For the other identities, since $(x)_{n}=\sum_{k=0}^{n}(-1)^{n-k}\left[\begin{array}{l}n \\ k\end{array}\right] x^{k}$ and $(x)_{n}$ is a sequence of binomial type [20,23], we obtain

$$
\left(\mathbf{w}_{\mathbf{x}}+r\right)_{n+r}=\sum_{j=0}^{n+r}\left(\begin{array}{c}
n+r \\
j
\end{array}\right)(r)_{j}\left(\mathbf{w}_{\mathbf{x}}\right)_{n+r-j}=(n+r) ! x^{n}(x+1)^{r} .
$$

So, the polynomials $x^{s} w_{n}(x ; r, s)$ and $(x+1)^{s} w_{n}(x, r, s)$ must be, respectively,

$$
\sum_{j=0}^{n}\left\{\begin{array}{c}
n+r \\
j+r
\end{array}\right\}_{r}\left(\mathbf{w}_{\mathbf{x}}\right)_{j+s}=\sum_{j=0}^{n}\left\{\begin{array}{c}
n+r \\
j+r
\end{array}\right\}_{r}\left(\mathbf{w}_{\mathbf{x}}-s\right)_{j}\left(\mathbf{w}_{\mathbf{x}}\right)_{s}=\left(\mathbf{w}_{\mathbf{x}}+r-s\right)^{n}\left(\mathbf{w}_{\mathbf{x}}\right)_{s},
$$


$\sum_{j=0}^{n}\left\{\begin{array}{l}n+r \\ j+r\end{array}\right\}_{r}\left(\mathbf{w}_{\mathbf{x}}+s\right)_{j+s}=\sum_{j=0}^{n}\left\{\begin{array}{l}n+r \\ j+r\end{array}\right\}_{r}\left(\mathbf{w}_{\mathbf{x}}\right)_{j}\left(\mathbf{w}_{\mathbf{x}}+s\right)_{s}=\left(\mathbf{w}_{\mathbf{x}}+r\right)^{n}\left(\mathbf{w}_{\mathbf{x}}+s\right)_{s}$.

The last two identities of Proposition 1 lead to:

Corollary 1. Let $r$; s be non-negative integers and $f$ be a polynomial. Then

$$
(x+1)^{s} f\left(\mathbf{w}_{\mathbf{x}}+r-s\right)\left(\mathbf{w}_{\mathbf{x}}\right)_{s}=x^{s} f\left(\mathbf{w}_{\mathbf{x}}+r\right)\left(\mathbf{w}_{\mathbf{x}}+s\right)_{s} .
$$

Proposition 2. Let $\mathcal{P}_{n}$ and $\mathcal{T}_{n}$ be the polynomials

$$
\mathcal{P}_{n}(x ; r)=\sum_{j=0}^{n}(-1)^{j}\left(\begin{array}{c}
j+r \\
r
\end{array}\right) x^{n-j} \text { and } \mathcal{T}_{n}(x ; r)=\sum_{j=0}^{n}\left(\begin{array}{c}
n+r \\
j+r
\end{array}\right) x^{j} .
$$

Then $\left(\mathbf{w}_{\mathbf{x}}-r-1\right)_{n}=n ! \mathcal{P}_{n}(x ; r)$ and $\left(\mathbf{w}_{\mathbf{x}}+n+r\right)_{n}=n ! \mathcal{T}_{n}(x ; r)$.

Proof. It suffices to observe that

$$
\begin{aligned}
\left(\mathbf{w}_{\mathbf{x}}-r-1\right)_{n} & =\sum_{j=0}^{n}\left(\begin{array}{l}
n \\
j
\end{array}\right)(-r-1)_{j}\left(\mathbf{w}_{\mathbf{x}}\right)_{n-j}=n ! \sum_{j=0}^{n}(-1)^{j}\left(\begin{array}{c}
j+r \\
r
\end{array}\right) x^{n-j}, \\
\left(\mathbf{w}_{\mathbf{x}}+n+r\right)_{n} & =\sum_{j=0}^{n}\left(\begin{array}{c}
n \\
j
\end{array}\right)(n+r)_{n-j}\left(\mathbf{w}_{\mathbf{x}}\right)_{j}=n ! \sum_{j=0}^{n}\left(\begin{array}{c}
n+r \\
j+r
\end{array}\right) x^{j} .
\end{aligned}
$$

The following theorem can be served to derive several identities and congruences for the $(r, s)$-geometric polynomials.

Theorem 1. Let $m, s$ be non-negative integers and $f$ be a polynomial. Then

$$
(x+1)^{m} f\left(\mathbf{w}_{\mathbf{x}}\right)-x^{m} f\left(\mathbf{w}_{\mathbf{x}}+m\right)=\sum_{k=0}^{m-1} f(k)(x+1)^{m-1-k} x^{k}, m \geq 1 .
$$

Proof. Set $f(x)=\sum_{k=0}^{n} a_{k} x^{k}$ and use Proposition 1 to obtain

$$
(x+1) f\left(\mathbf{w}_{\mathbf{x}}\right)-x f\left(\mathbf{w}_{\mathbf{x}}+1\right)=f(0)+\sum_{k=0}^{n} a_{k}\left((x+1) \mathbf{w}_{\mathbf{x}}^{k}-x\left(\mathbf{w}_{\mathbf{x}}+1\right)^{k}\right)=f(0) .
$$

So, the identity is true for $m=1$. Assume it is true for $m$. Then

$$
\begin{aligned}
(x+1)^{m+1} f\left(\mathbf{w}_{\mathbf{x}}\right) & =(x+1)\left(\sum_{k=0}^{m-1}(x+1)^{m-1-k} x^{k} f(k)+x^{m} f\left(\mathbf{w}_{\mathbf{x}}+m\right)\right) \\
& =\sum_{k=0}^{m-1}(x+1)^{m-k} x^{k} f(k)+x^{m}(x+1) f\left(\mathbf{w}_{\mathbf{x}}+m\right)
\end{aligned}
$$


and since $(x+1) f\left(\mathbf{w}_{\mathbf{x}}+m\right)-x f\left(\mathbf{w}_{\mathbf{x}}+m+1\right)=f(m)$, we can write

$$
\begin{aligned}
(x+1)^{m+1} f\left(\mathbf{w}_{\mathbf{x}}\right) & =\sum_{k=0}^{m-1}(x+1)^{m-k} x^{k} f(k)+x^{m}\left(x f\left(\mathbf{w}_{\mathbf{x}}+m+1\right)+f(m)\right) \\
& =\sum_{k=0}^{m-1}(x+1)^{m-k} x^{k} f(k)+x^{m} f(m)+x^{m+1} f\left(\mathbf{w}_{\mathbf{x}}+m+1\right) \\
& =\sum_{k=0}^{m}(x+1)^{m-k} x^{k} f(k)+x^{m+1} f\left(\mathbf{w}_{\mathbf{x}}+m+1\right)
\end{aligned}
$$

which concludes the induction step.

We note that for $f(x)=x^{n}$ and $x=1$ in Theorem 1 we obtain Proposition 3.3 given in [8].

Corollary 2. For any polynomial $f$ there holds

$$
f\left(\mathbf{w}_{\mathbf{x}}\right)=\frac{1}{1+x} \sum_{k \geq 0} f(k)\left(\frac{x}{1+x}\right)^{k}, x>-\frac{1}{2} .
$$

Proof. For $m=1$ in Theorem 1, when we replace $f(x)$ by $f(x+r)$ we get the identity $f(r)=(x+1) f\left(\mathbf{w}_{\mathbf{x}}+r\right)-x f\left(\mathbf{w}_{\mathbf{x}}+r+1\right)$. Then

$$
\begin{aligned}
\text { RHS } & =\lim _{n \rightarrow \infty} \frac{1}{1+x} \sum_{k=0}^{n}\left(\frac{x}{1+x}\right)^{k}\left((x+1) f\left(\mathbf{w}_{\mathbf{x}}+k\right)-x f\left(\mathbf{w}_{\mathbf{x}}+k+1\right)\right) \\
& =\lim _{n \rightarrow \infty}\left(f\left(\mathbf{w}_{\mathbf{x}}\right)-\left(\frac{x}{1+x}\right)^{n+1} f\left(\mathbf{w}_{\mathbf{x}}+n+1\right)\right)=f\left(\mathbf{w}_{\mathbf{x}}\right)
\end{aligned}
$$

which completes the proof.

Corollary 3. Let $n, r$, s be non-negative integers.

For $f(x)=(x+r)^{n}(x+s)_{s}$ or $(x+r-s)^{n}(x)_{s}$ in Corollary 2 we obtain

$$
w_{n}(x ; r, s)=\frac{s !}{(1+x)^{s+1}} \sum_{k \geq 0}\left(\begin{array}{c}
k+s \\
s
\end{array}\right)(k+r)^{n}\left(\frac{x}{1+x}\right)^{k}, x>-\frac{1}{2} .
$$

Corollary 4. For any integers $r \geq 0, s \geq 0$ and $n \geq 1$ the polynomial $w_{n}(x, r, s+$ $r)$ has only real non-positive zeros.

Proof. From Corollary 3 we may state

$$
x^{r}(x+1)^{s} w_{n+1}(x ; r, s+r)=x \frac{d}{d x}\left(x^{r}(x+1)^{s+1} w_{n}(x ; r, s+r)\right)
$$


and using the recurrence relation of $r$-Stirling numbers we conclude that this identity remains true for all real number $x$. So, by induction on $n$, it follows that $w_{n}(x ; r, s+$ $r), n \geq 1$, has only real non-positive zeros.

Lemma 1. For any non-negative integers $n \geq 2$ there holds

$$
(1+x) w_{n-1}(x)=\sum_{k=1}^{n}\left\{\begin{array}{l}
n \\
k
\end{array}\right\}(k-1) ! x^{k} .
$$

Proof. From the definition of geometric polynomials, we have

$$
\begin{aligned}
(1+x) w_{n-1}(x) & =\sum_{k=1}^{n-1}\left\{\begin{array}{c}
n-1 \\
k
\end{array}\right\} k ! x^{k}+\sum_{k=1}^{n-1}\left\{\begin{array}{c}
n-1 \\
k
\end{array}\right\} k ! x^{k+1} \\
& =\sum_{k=1}^{n}\left(k\left\{\begin{array}{c}
n-1 \\
k
\end{array}\right\}+\left\{\begin{array}{l}
n-1 \\
k-1
\end{array}\right\}\right)(k-1) ! x^{k} \\
& =\sum_{k=1}^{n}\left\{\begin{array}{l}
n \\
k
\end{array}\right\}(k-1) ! x^{k} .
\end{aligned}
$$

For more explicit formulae for geometric polynomials, see for example [15].

Proposition 3. Let $n, r, s$ be non-negative integers. Then

$$
\log \left(1+\sum_{n \geq 1} \frac{w_{n}(x ; r, s)}{s !} \frac{t^{n}}{n !}\right)=(r+(s+1) x) t+(s+1)(x+1) \sum_{n \geq 2} w_{n-1}(x) \frac{t^{n}}{n !} .
$$

In particular, for $r=s=0$ we get

$$
\log \left(1+\sum_{n \geq 1} w_{n}(x) \frac{t^{n}}{n !}\right)=x t+(x+1) \sum_{n \geq 2} w_{n-1}(x) \frac{t^{n}}{n !} .
$$

Proof. One can verify easily that the exponential generating function of the polynomials $w_{n}(x ; r, s)$ is to be $s ! \exp (r t)(1-x(\exp (t)-1))^{-s-1}$. Then, upon using this generating function and the last Lemma, we can write

$$
\begin{aligned}
L H S & =r t-(s+1) \ln (1-x(\exp (t)-1)) \\
& =r t+(s+1) \sum_{k \geq 1} \frac{x^{k}}{k}(\exp (t)-1)^{k} \\
& =r t+(s+1) \sum_{k \geq 1}(k-1) ! x^{k} \sum_{n \geq k}\left\{\begin{array}{l}
n \\
k
\end{array}\right\} \frac{t^{n}}{n !}
\end{aligned}
$$




$$
\begin{aligned}
& =r t+(s+1) x t+(s+1) \sum_{n \geq 2} \frac{t^{n}}{n !} \sum_{k=1}^{n}\left\{\begin{array}{l}
n \\
k
\end{array}\right\}(k-1) ! x^{k} \\
& =(r+(s+1) x) t+(s+1)(x+1) \sum_{n \geq 2} w_{n-1}(x) \frac{t^{n}}{n !} .
\end{aligned}
$$

\section{CONGRUENCES INVOLVING THE $(\mathrm{R}, \mathrm{S})$-GeOMETRIC POLYNOMIALS}

In this section, we give some congruences involving the $(r, s)$-geometric polynomials. Let $\mathbb{Z}_{p}$ be the ring of $p$-adic integers and for two polynomials $f(x), g(x) \in$ $\mathbb{Z}_{p}[x]$, the congruence $f(x) \equiv g(x)\left(\bmod p \mathbb{Z}_{p}[x]\right)$ means that the corresponding coefficients of $f(x)$ and $g(x)$ are congruent modulo $p$. This congruence will be used later as $f(x) \equiv g(x)$ and we will use $a \equiv b$ instead $a \equiv b(\bmod p)$.

Proposition 4. Let $n, r, s$ be non-negative integers and $p$ be a prime number. Then, for any polynomial $f$ with integer coefficients there holds

$$
\sum_{k=0}^{p-1} f(k)(x+1)^{p-1-k} x^{k} \equiv f\left(\mathbf{w}_{\mathbf{x}}\right)
$$

In particular, for $f(x)=(x+r-s)^{n}(x)_{s}$ or $(x+r)^{n}(x+s)_{s}$ we get, respectively,

$$
\begin{aligned}
& \sum_{k=0}^{p-1}(r-s+k)^{n}(k)_{s}(x+1)^{p-1-k} x^{k} \equiv x^{s} w_{n}(x ; r, s), \\
& \sum_{k=0}^{p-1}(r+k)^{n}(s+k)_{s}(x+1)^{p-1-k} x^{k} \equiv(x+1)^{s} w_{n}(x ; r, s) .
\end{aligned}
$$

Proof. For $m=p$ be a prime number, Theorem 1 implies

$$
L H S=(x+1)^{p} f\left(\mathbf{w}_{\mathbf{x}}\right)-x^{p} f\left(\mathbf{w}_{\mathbf{x}}+p\right) \equiv\left(x^{p}+1\right) f\left(\mathbf{w}_{\mathbf{x}}\right)-x^{p} f\left(\mathbf{w}_{\mathbf{x}}\right)=f\left(\mathbf{w}_{\mathbf{x}}\right) .
$$

For the particular cases, use Proposition 1.

Corollary 5. Let $n, r, s, m, q$ be non-negative integers and $p$ be a prime number. Then, for any polynomials $f$ and $g$ with integer coefficients there holds

$$
\left(f\left(\mathbf{w}_{\mathbf{x}}\right)\right)^{p} g\left(\mathbf{w}_{\mathbf{x}}\right) \equiv f\left(\mathbf{w}_{\mathbf{x}}\right) g\left(\mathbf{w}_{\mathbf{x}}\right) .
$$

In particular, we have $w_{m p+q}(x ; r, s) \equiv w_{m+q}(x ; r, s)$.

Proof. By Fermat's little theorem and by twice application of Proposition 4 we may state

$L H S \equiv \sum_{k=0}^{p-1}(f(k))^{p} g(k)(x+1)^{p-1-k} x^{k} \equiv \sum_{k=0}^{p-1} f(k) g(k)(x+1)^{p-1-k} x^{k}=R H S$. 
We note that, for $f(x)=x^{m}, g(x)=x^{q}$ and $x=1$, Corollary 5 may be seen as a particular case of Theorem 3.1 given in [8].

Corollary 6. For any non-negative integers $m \geq 1, n, r, s$ and any prime number $p$, there hold

$$
\begin{aligned}
& (x+1)^{s+1}\left(w_{m(p-1)}(x ; r, s)-s !\right) \equiv-\left(s-r^{\prime}\right)_{s}(x+1)^{r^{\prime}} x^{p-r^{\prime}}, r^{\prime} \neq 0, \\
& (x+1)^{s+1}\left(w_{m(p-1)}(x ; r, s)-s !\right) \equiv-s !\left(x^{p}+1\right), r^{\prime}=0,
\end{aligned}
$$

where $r^{\prime} \equiv r$ and $r^{\prime} \in\{0,1, \ldots, p-1\}$.

Proof. Set $n=m(p-1)$ in Proposition 4. If $r^{\prime} \neq 0$ we get

$$
\begin{aligned}
(x+1)^{s} w_{m(p-1)}(x ; r, s) \equiv & \sum_{k=0}^{p-1}\left(r^{\prime}+k\right)^{m(p-1)}(s+k)_{s}(x+1)^{p-1-k} x^{k} \\
\equiv & \sum_{k=0, r^{\prime}+k \neq p}^{p-1}(s+k)_{s}(x+1)^{p-1-k} x^{k} \\
= & \sum_{k=0}^{p-1}(s+k)_{s}(x+1)^{p-1-k} x^{k} \\
& -\left(s-r^{\prime}+p\right)_{s}(x+1)^{r^{\prime}-1} x^{p-r^{\prime}} \\
\equiv & (x+1)^{s} w_{0}(x ; 0, s)-\left(s-r^{\prime}\right)_{s}(x+1)^{r^{\prime}-1} x^{p-r^{\prime}} \\
\equiv & s !(x+1)^{s}-\left(s-r^{\prime}\right)_{s}(x+1)^{r^{\prime}-1} x^{p-r^{\prime}}
\end{aligned}
$$

and if $r^{\prime}=0$ we get

$$
\begin{aligned}
(x+1)^{s+1} w_{m(p-1)}(x ; r, s) & \equiv \sum_{k=1}^{p-1}(s+k)_{s}(x+1)^{p-k} x^{k} \\
& =\sum_{k=0}^{p-1}(s+k)_{s}(x+1)^{p-k} x^{k}-s !(x+1)^{p} \\
& =(x+1)^{s+1} w_{0}(x ; 0, s)-s !(x+1)^{p} \\
& =s !(x+1)^{s+1}-s !\left(x^{p}+1\right) .
\end{aligned}
$$

which complete the proof.

Remark 1. For $r=s=m-1=0$ in Corollary 6 or $n=p$ in Lemma 1 we obtain $(x+1) w_{p-1}(x) \equiv x-x^{p}$ which gives for $x=1$ the known congruence $w_{p-1} \equiv 0$, see [8]. 
Now, we give some curious congruences on $(r, s)$-geometric polynomials and on $\left(r_{1}, \ldots, r_{q}\right)$-geometric polynomials defined below.

Theorem 2. For any integers $n, m, r, s \geq 0$ and any prime number $p \nmid m$, there holds

$$
\sum_{k=1}^{p-1} \frac{w_{n+k}(x ; r, s)}{(-m)^{k}} \equiv(-m)^{n}\left(w_{p-1}(x ; r+m, s)-s !\right) .
$$

Proof. Upon using the identity $x^{s} w_{n}(x ; r, s)=\left(\mathbf{w}_{\mathbf{x}}+r-s\right)^{n}\left(\mathbf{w}_{\mathbf{x}}\right)_{s}$ and the known congruence $(-m)^{-k} \equiv\left(\begin{array}{c}p-1 \\ k\end{array}\right) m^{p-1-k}$ we obtain

$$
\begin{aligned}
x^{s} L H S & \equiv \sum_{k=0}^{p-1}\left(\begin{array}{c}
p-1 \\
k
\end{array}\right) m^{p-1-k}\left(\mathbf{w}_{\mathbf{x}}+r-s\right)^{n+k}\left(\mathbf{w}_{\mathbf{x}}\right)_{s} \\
& =\left(\mathbf{w}_{\mathbf{x}}+r-s\right)^{n}\left(\mathbf{w}_{\mathbf{x}}+r+m-s\right)^{p-1}\left(\mathbf{w}_{\mathbf{x}}\right)_{s} \\
& =\sum_{j=0}^{n}\left(\begin{array}{c}
n \\
j
\end{array}\right)(-m)^{n-j}\left(\mathbf{w}_{\mathbf{x}}+r+m-s\right)^{j+p-1}\left(\mathbf{w}_{\mathbf{x}}\right)_{s} \\
& =(-m)^{n}\left(\mathbf{w}_{\mathbf{x}}+r+m-s\right)^{p-1}\left(\mathbf{w}_{\mathbf{x}}\right)_{s} \\
& +\delta_{(n \geq 1)} \sum_{j=1}^{n}\left(\begin{array}{c}
n \\
j
\end{array}\right)(-m)^{n-j}\left(\mathbf{w}_{\mathbf{x}}+r+m-s\right)^{j+p-1}\left(\mathbf{w}_{\mathbf{x}}\right)_{s} \\
& =x^{s}(-m)^{n} w_{p-1}(x ; r+m, s) \\
& +\delta_{(n \geq 1)} x^{s} \sum_{j=1}^{n}\left(\begin{array}{c}
n \\
j
\end{array}\right)(-m)^{n-j} w_{p+j-1}(x ; r+m, s) \\
& \equiv x^{s}(-m)^{n} w_{p-1}(x ; r+m, s) \\
& +\delta_{(n \geq 1)} x^{s} \sum_{j=1}^{n}\left(\begin{array}{c}
n \\
j
\end{array}\right)(-m)^{n-j} w_{j}(x ; r+m, s) \\
& =x^{s}(-m)^{n} w_{p-1}(x ; r+m, s)+\delta_{(n \geq 1)} x^{s}\left(w_{n}(x ; r, s)-(-m)^{n} s !\right) \\
& =x^{s}\left[(-m)^{n} w_{p-1}(x ; r+m, s)+w_{n}(x ; r, s)-(-m)^{n} s !\right],
\end{aligned}
$$

where $\delta$ is the Kronecker's symbol, i.e. $\delta_{(n \geq 1)}=1$ if $n \geq 1$ and 0 otherwise.

Let $\mathbf{r}_{q}=\left(r_{1}, \ldots, r_{q}\right)$ be a vector of non-negative integers and let

$$
w_{n}\left(x ; \mathbf{r}_{q}\right)=\sum_{j=0}^{n+\left|\mathbf{r}_{q-1}\right|}\left\{\begin{array}{c}
n+\left|\mathbf{r}_{q}\right| \\
j+r_{q}
\end{array}\right\}_{\mathbf{r}_{q}}\left(j+r_{q}\right) ! x^{j}, 0 \leq r_{1} \leq \cdots \leq r_{q},
$$


where $\left\{\begin{array}{c}n+\left|\mathbf{r}_{q}\right| \\ j+r_{q}\end{array}\right\}_{\mathbf{r}_{q}}$ are the $\left(r_{1}, \ldots, r_{q}\right)$-Stirling numbers defined by Mihoubi et al. [21]. This polynomial is a generalization of the $r$-geometric polynomials $w_{n}(x ; r):=$ $w_{n}(x ; r, r)$.

Proposition 5. For any non-negative integers $n, m$ and any prime $p \nmid m$, there holds

$$
x^{r_{q}} \sum_{k=1}^{p-1} \frac{w_{n+k}\left(x ; \mathbf{r}_{q}\right)}{(-m)^{k}} \equiv(-m)^{n}(-m)_{r_{1}} \cdots(-m)_{r_{q}}\left(w_{p-1}(x ; m, 0)-1\right) .
$$

In particular, for $q=1$ and $r_{q}=r$ we obtain

$$
x^{r} \sum_{k=1}^{p-1} \frac{w_{n+k}(x ; r, r)}{(-m)^{k}} \equiv(-m)^{n}(-m)_{r}\left(w_{p-1}(x ; m, 0)-1\right) .
$$

Proof. By the identity $\left(\mathbf{w}_{\mathbf{x}}\right)_{n}=n ! x^{n}$ and by [21, Th. 10] we have

$$
\begin{aligned}
x^{r_{q}} w_{n}\left(x ; \mathbf{r}_{q}\right) & =\sum_{j=0}^{n+\left|\mathbf{r}_{q-1}\right|}\left\{\begin{array}{c}
n+\left|\mathbf{r}_{q}\right| \\
j+r_{q}
\end{array}\right\}_{\mathbf{r}_{q}}\left(\mathbf{w}_{\mathbf{x}}\right)_{j+r_{q}} \\
& =\sum_{j=0}^{n+\left|\mathbf{r}_{q-1}\right|}\left\{\begin{array}{c}
n+\left|\mathbf{r}_{q}\right| \\
j+r_{q}
\end{array}\right\}_{\mathbf{r}_{q}}\left(\mathbf{w}_{\mathbf{x}}-r_{q}\right)_{j}\left(\mathbf{w}_{\mathbf{x}}\right)_{r_{q}} \\
& =\mathbf{w}_{\mathbf{x}}^{n}\left(\mathbf{w}_{\mathbf{x}}\right)_{r_{1}} \cdots\left(\mathbf{w}_{\mathbf{x}}\right)_{r_{q}} \\
& =\sum_{k=0}^{\left|\mathbf{r}_{q}\right|} a_{k}\left(\mathbf{r}_{q}\right) \mathbf{w}_{\mathbf{x}}^{n+k} \\
& =\sum_{j=0}^{\left|\mathbf{r}_{q}\right|} a_{j}\left(\mathbf{r}_{q}\right) w_{n+j}(x),
\end{aligned}
$$

where $\sum_{k=0}^{\left|\mathbf{r}_{q}\right|} a_{k}\left(\mathbf{r}_{q}\right) u^{k}=(u)_{r_{1}} \cdots(u)_{r_{q}}$. So, by application of Theorem 2 we get

$$
\begin{aligned}
x^{r_{q}} \sum_{k=1}^{p-1} \frac{w_{n+k}\left(x ; \mathbf{r}_{q}\right)}{(-m)^{k}} & =\sum_{j=0}^{\left|\mathbf{r}_{q}\right|} a_{j}\left(\mathbf{r}_{q}\right) \sum_{k=1}^{p-1} \frac{w_{n+j+k}(x ; 0,0)}{(-m)^{k}} \\
& \equiv \sum_{j=0}^{\left|\mathbf{r}_{q}\right|} a_{j}\left(\mathbf{r}_{q}\right)(-m)^{n+j}\left(w_{p-1}(x ; m, 0)-1\right) \\
& =(-m)^{n}(-m)_{r_{1}} \cdots(-m)_{r_{q}}\left(w_{p-1}(x ; m, 0)-1\right) .
\end{aligned}
$$


Remark 2. Since $x^{r_{q}} w_{n}\left(x ; \mathbf{r}_{q}\right)=\mathbf{w}_{\mathbf{x}}^{n}\left(\mathbf{w}_{\mathbf{x}}\right)_{r_{1}} \cdots\left(\mathbf{w}_{\mathbf{x}}\right)_{r_{q}}$, then, for $g(x)=x^{q}(x)_{r_{1}} \cdots(x)_{r_{q}}$ and $f(x)=x^{m}$ in Corollary 5 we obtain

$$
\begin{gathered}
w_{m p+q}\left(x ; \mathbf{r}_{q}\right) \equiv w_{m+q}\left(x ; \mathbf{r}_{q}\right), \\
w_{m(p-1)}\left(x ; \mathbf{r}_{q}\right) \equiv w_{0}\left(x ; \mathbf{r}_{q}\right), r_{1} \cdots r_{q} \neq 0, m \geq 0 .
\end{gathered}
$$

Corollary 7. Let $a_{0}(x), \ldots, a_{t}(x)$ be polynomials with integer coefficients,

$$
\mathcal{R}_{n, t}(x ; r, s)=\sum_{i=0}^{t} a_{i}(x) w_{n+i}(x ; r, s) \text { and } \mathscr{L}_{t}(x, y)=\sum_{i=0}^{t} a_{i}(x) y^{i} .
$$

Then, for any non-negative integers $n, m, r, s$ and any prime $p \nmid m$, there hold

$$
\sum_{k=1}^{p-1} \frac{\mathcal{R}_{n+k, t}(x ; r, s)}{(-m)^{k}} \equiv(-m)^{n} \mathscr{L}_{t}(x,-m)\left(w_{p-1}(x ; r+m, s)-s !\right) .
$$

Proof. Theorem 2 implies

$$
\begin{aligned}
\sum_{k=1}^{p-1} \frac{\mathcal{R}_{n+k, t}(x ; r, s)}{(-m)^{k}} & =\sum_{j=0}^{t} a_{j}(x) \sum_{k=1}^{p-1} \frac{w_{n+k+j}(x ; r, s)}{(-m)^{k}} \\
& \equiv \sum_{j=0}^{t} a_{j}(x)(-m)^{n+j}\left(w_{p-1}(x ; r+m, s)-s !\right) \\
& =(-m)^{n} \mathscr{L}_{t}(x,-m)\left(w_{p-1}(x ; r+m, s)-s !\right) .
\end{aligned}
$$

4. Congruences Involving $w_{n}(x ; r, s), \mathcal{P}_{n}(x, r)$ AND $\mathcal{T}_{n}(x, r)$

The following theorem gives connection in congruences between the polynomials $w_{n}$ and $\mathcal{P}_{n}$.

Theorem 3. Let $n, r$ be non-negative integers and $p$ be a prime number. Then, for $m \in\{0, \ldots, p-1\}$ there holds

$$
\sum_{k=m}^{p-1}(-x)^{k} \frac{w_{n}(x ; r+k, k)}{(k-m) !} \equiv(-1)^{m} m !(r+m)^{n} \mathcal{P}_{p-1}(x, m) .
$$

In particular, for $m=0$, we get

$$
\sum_{k=0}^{p-1}(-x)^{k} \frac{w_{n}(x ; r+k, k)}{k !} \equiv r^{n}\left(1+x+\cdots+x^{p-1}\right) .
$$


Proof. For $k<m$ we get $\langle m+1\rangle_{p-1-k}=0$ and for $m \leq k \leq p-1$ we have

$$
\langle m+1\rangle_{p-1-k}=\frac{(m+p-k-1) !}{m !}=\frac{(p-1-(k-m)) !}{m !} \equiv-\frac{1}{m !} \frac{(-1)^{k-m}}{(k-m) !} .
$$

where $\langle x\rangle_{n}=x(x+1) \cdots(x+n-1)$ if $n \geq 1$ and $\langle x\rangle_{0}=1$. Then

$$
\begin{aligned}
L H S & \equiv-(-1)^{m} m ! \sum_{k=0}^{p-1}\langle m+1\rangle_{p-1-k} x^{k} w_{n}(x ; r+k, k) \\
& \equiv-(-1)^{m} m ! \sum_{k=0}^{p-1}\langle m-p+1\rangle_{p-1-k}\left(\mathbf{w}_{\mathbf{x}}+r\right)^{n}\left(\mathbf{w}_{\mathbf{x}}\right)_{k} \\
& \equiv-(-1)^{m} m ! \sum_{k=0}^{p-1}\left(\begin{array}{c}
p-1 \\
k
\end{array}\right)\langle m-p+1\rangle_{p-1-k}\left(\mathbf{w}_{\mathbf{x}}+r\right)^{n}\left\langle-\mathbf{w}_{\mathbf{x}}\right\rangle_{k} \\
& =-(-1)^{m} m !\left\langle m-p+1-\mathbf{w}_{\mathbf{x}}\right\rangle_{p-1}\left(\mathbf{w}_{\mathbf{x}}+r\right)^{n} \\
& =-(-1)^{m} m !\left(\mathbf{w}_{\mathbf{x}}-m+p-1\right)_{p-1}\left(\mathbf{w}_{\mathbf{x}}+r\right)^{n} \\
& =-(-1)^{m} m !\left(\mathbf{w}_{\mathbf{x}}-m+r+m\right)^{n}\left(\mathbf{w}_{\mathbf{x}}-m+p-1\right)_{p-1} \\
& =-(-1)^{m} m ! \sum_{j=0}^{n}\left\{\begin{array}{c}
n+r+m \\
j+r+m
\end{array}\right\}_{r+m} \quad\left(\mathbf{w}_{\mathbf{x}}-m\right)_{j}\left(\mathbf{w}_{\mathbf{x}}-m+p-1\right)_{p-1} .
\end{aligned}
$$

But for $j \geq 1$ we have

$$
\begin{aligned}
& \left(\mathbf{w}_{\mathbf{x}}-m\right)_{j}\left(\mathbf{w}_{\mathbf{x}}-m+p-1\right)_{p-1}=\left(\mathbf{w}_{\mathbf{x}}-m+p-1\right)_{j+p-1} \\
& \equiv\left(\mathbf{w}_{\mathbf{x}}-m-1\right)_{j+p-1}=(j+p-1) ! \mathcal{P}_{j+p-1}(x, m+1) \\
& \equiv-\delta_{(j=0)} \mathcal{P}_{p-1}(x, m+1)
\end{aligned}
$$

hence, it follows $L H S \equiv(-1)^{m} m !(r+m)^{n} \mathcal{P}_{p-1}(x, m)$.

A connection in congruences between the polynomials $w_{n}$ and $\mathcal{T}_{n}$ is to be:

Theorem 4. For any integers $n, m, r \geq 0$ and any prime $p$, there holds

$$
\sum_{k=0}^{p-1}(-m)_{p-1-k}(x+1)^{k} w_{n}(x ; r+m, k) \equiv-r^{n} \mathcal{T}_{p-1}(x ; m) .
$$

Proof. Upon using the identity $(x+1)^{s} w_{n}(x ; r, s)=\left(\mathbf{w}_{\mathbf{x}}+r\right)^{n}\left(\mathbf{w}_{\mathbf{x}}+s\right)_{s}$ and the known congruence $(m)_{p-1-k} \equiv\left(\begin{array}{c}p-1 \\ k\end{array}\right)\langle-m\rangle_{p-1-k}$ we obtain

$$
L H S \equiv \sum_{k=0}^{p-1}\left(\begin{array}{c}
p-1 \\
k
\end{array}\right)\langle m\rangle_{p-1-k}\left(\mathbf{w}_{\mathbf{x}}+r+m\right)^{n}\left(\mathbf{w}_{\mathbf{x}}+k\right)_{k}
$$




$$
\begin{aligned}
& \equiv \sum_{k=0}^{p-1}\left(\begin{array}{c}
p-1 \\
k
\end{array}\right)\langle m\rangle_{p-1-k}\left(\mathbf{w}_{\mathbf{x}}+r+m\right)^{n}\left\langle\mathbf{w}_{\mathbf{x}}+1\right\rangle_{k} \\
&=\left(\mathbf{w}_{\mathbf{x}}+r+m\right)^{n}\left\langle\mathbf{w}_{\mathbf{x}}+m+1\right\rangle_{p-1} \\
& \equiv\left(\mathbf{w}_{\mathbf{x}}+m+r\right)^{n}\left(\mathbf{w}_{\mathbf{x}}+m+p-1\right)_{p-1} \\
&= \sum_{j=0}^{n}\left\{\begin{array}{c}
n+r \\
j+r
\end{array}\right\}_{r}\left(\mathbf{w}_{\mathbf{x}}+m\right)_{j}\left(\mathbf{w}_{\mathbf{x}}+m+p-1\right)_{p-1} \\
&= \sum_{j=0}^{n}\left\{\begin{array}{c}
n+r \\
j+r
\end{array}\right\}_{r}\left(\mathbf{w}_{\mathbf{x}}+m+p-1\right)_{j+p-1} \\
&= \sum_{j=0}^{n}\left\{\begin{array}{c}
n+r \\
j+r
\end{array}\right\}_{r}(j+p-1) ! \mathcal{T}_{j+p-1}(x ; m-j) \\
&=(p-1) ! \mathcal{T}_{p-1}(x ; m)+\sum_{j=1}^{n}\left\{\begin{array}{c}
n+r \\
j+r
\end{array}\right\}_{r}(j+p-1) ! \mathcal{T}_{j+p-1}(x ; m-j) \\
& \equiv-r^{n} \mathcal{T}_{p-1}(x ; m) .
\end{aligned}
$$

Corollary 8. Let $\mathcal{R}_{n, t}(x ; r, s)$ be as in Corollary 7. Then, for any non-negative integers $n, m, r, s$ and any prime $p \nmid m$, there holds

$$
\sum_{k=m}^{p-1}(-x)^{k}\left(\begin{array}{l}
k \\
m
\end{array}\right) \frac{\mathcal{R}_{n, t}(x ; r+k, k)}{k !} \equiv(-1)^{m}(r+m)^{n} \mathscr{L}_{t}(x, r+m) \mathcal{P}_{p-1}(x, m) .
$$

Proof. Theorem 3 implies

$$
\begin{aligned}
L H S & =\sum_{j=0}^{t} a_{j}(x) \sum_{k=m}^{p-1}(-x)^{k}\left(\begin{array}{l}
k \\
m
\end{array}\right) \frac{w_{n+j}(x ; r+k, k)}{k !} \\
& \equiv \sum_{j=0}^{t} a_{j}(x)(-1)^{m}(r+m)^{n+j} \mathcal{P}_{p-1}(x, m) \\
& \equiv(-1)^{m}(r+m)^{n} \mathscr{L}_{t}(x, r+m) \mathcal{P}_{p-1}(x, m) .
\end{aligned}
$$

\section{REFERENCES}

[1] A. Benyattou and M. Mihoubi, "Curious congruences related to the Bell polynomials," Quaest Math., vol. 40, pp. 1-12, 2017, doi: 10.2989/16073606.2017.1391349.

[2] K. N. Boyadzhiev, "A series transformation formula and related polynomials," Int. J. Math. Math. Sci., vol. 2005, no. 23, pp. 3849-3866, 2005, doi: 10.1155/IJMMS.2005.3849. 
[3] K. N. Boyadzhiev and A. Dil, "Geometric polynomials: properties and applications to series with zeta values," Analysis Math., vol. 42, no. 3, pp. 203-224, 2016, doi: 10.1007/s10476-016-0302-y.

[4] A. Z. Broder, "The r-Stirling numbers," Discrete Math., vol. 49, no. 3, pp. 241-259, 1984, doi: 10.1016/0012-365X(84)90161-4.

[5] A. D. Bucchianico and D. Loeb, "A selected survey of umbral calculus," Electron. J. Combin., vol. 2, pp. 1-34, 2000.

[6] M. B. Can and M. Joyce, "Ordered Bell numbers, Hermite polynomials, skew Young tableaux, and Borel orbits," J. Comb. Theory Ser. A, vol. 119, no. 8, pp. 1798-1810, 2012, doi: 10.1016/j.jcta.2012.06.002.

[7] M. E. Dasef and S. M. Kautz, "Some sums of some importance," College Math. J., vol. 28, pp. 52-55, 1997.

[8] T. Diagana and H. Maïga, "Some new identities and congruences for Fubini numbers," J. Number Theory, vol. 173, pp. 547-569, 2017.

[9] A. Dil and V. Kurt, "Investigating geometric and exponential polynomials with Euler-Seidel matrices," J. Integer Seq., vol. 14, no. 4, 2011.

[10] A. Dil and V. Kurt, "Polynomials related to harmonic numbers and evaluation of harmonic number series II," Appl. Anal. Discrete Math., vol. 5, pp. 212-229, 2011.

[11] D. Dumont, "Matrices d'Euler-Siedel," Sémin. Lothar. Comb., vol. 5, 1981.

[12] P. Flajolet and R. Sedgewick, Analytic combinatorics. Cambridge university press, 2009.

[13] I. M. Gessel, "Applications of the classical umbral calculus," Algebra Universalis, vol. 49, no. 4, pp. 397-434, 2003, doi: 10.1007/s00012-003-1813-5.

[14] R. D. James, "The factors of a square-free integer," Canad. Math. Bull., vol. 11, pp. 733-735, 1968, doi: 10.4153/CMB-1968-089-7.

[15] L. Kargin, "Some formulae for products of geometric polynomials with applications," J. Integer Seq., vol. 20, no. 2, 2017.

[16] L. Kargin and B. Çekim, "Higher order generalized geometric polynomials," Turk. J. Math., vol. 42, pp. 887-903, 2018.

[17] L. Kargin and R. B. Corcino, "Generalization of Mellin derivative and its applications," Integral Transforms Spec. Funct., vol. 27, pp. 620-631, 2016, doi: 10.1080/10652469.2016.1174701.

[18] I. Mező, "Periodicity of the last digits of some combinatorial sequences," J. Integer Seq., vol. 17, no. $1,2014$.

[19] I. Mező and J. L. Ramírez, "Divisibility properties of the r-Bell numbers and polynomials," J. Number Theory, vol. 177, pp. 136-152, 2017, doi: 10.1016/j.jnt.2017.01.022.

[20] M. Mihoubi, "Bell polynomials and binomial type sequences," Discrete Math., vol. 308, no. 12, pp. 2450-2459, 2008, doi: 10.1016/j.disc.2007.05.010.

[21] M. Mihoubi and M. S. Maamra, "The (r1,.., rp)-Stirling numbers of the second kind," Integers, vol. 12, no. 5, pp. 1047-1059, 2012, doi: 10.1515/integers-2012-0022.

[22] T. J. Robinson, "Formal calculus and umbral calculus," Electron. J. Combin., vol. 17, no. 1, p. R95, 2010

[23] S. Roman, The Umbral Calculus. Courier Corporation, 2013.

[24] S. M. Roman and G.-C. Rota, "The umbral calculus," Adv. Math., vol. 27, no. 2, pp. 95-188, 1978, doi: 10.1016/0001-8708(78)90087-7.

[25] G. C. Rota and B. D. Taylor, “The classical umbral calculus," SIAM J. Math. Anal., vol. 25, no. 2, pp. 694-711, 1994, doi: 10.1137/S0036141093245616.

[26] R. P. Stanley, Enumerative Combinatorics I. Cambridge University press,, 1997.

[27] Y. Sun, X. Wu, and J. Zhuang, "Congruences on the Bell polynomials and the derangement polynomials," J. Number Theory, vol. 133, no. 5, pp. 1564-1571, 2013, doi: 10.1016/j.jnt.2012.08.031.

[28] S. M. Tanny, "On some numbers related to the Bell numbers," Canad. Math. Bull., vol. 17, no. 5, p. 733,1975 , doi: 10.4153/CMB-1974-132-8. 
[29] D. J. Velleman and G. S. Call, "Permutations and combination locks," Math. Mag., vol. 68, no. 4, pp. 243-253, 1995, doi: 10.2307/2690567.

Authors' addresses

Miloud Mihoubi

USTHB, Faculty of Mathematics, RECITS Laboratory, P. O. Box 32 El Alia 16111 Algiers, Algeria

E-mail address: mmihoubi@usthb.dz, miloudmihoubi@gmail.com

Said Taharbouchet

USTHB, Faculty of Mathematics, RECITS Laboratory, P. O. Box 32 El Alia 16111 Algiers, Algeria

E-mail address: staharbouchet@usthb.dz, said.taharbouchet@gmail.com 